\title{
A Search for Dyson Spheres Around Late-type Stars in the Solar Neighborhood
}

\author{
Jun Jugaku \\ Research Institute of Civilization, 2-29-3 Sakuragaoka, Tama-shi, Tokyo \\ 206-0013, Japan \\ Shiro Nishimura \\ National Astronomical Observatory, Mitaka, Tokyo 181-8588, Japan
}

\begin{abstract}
We continued our search for partial (incomplete) Dyson spheres associated with solar-type stars (spectral types F, G and $\mathrm{K}$ ) within $25 \mathrm{pc}$ of the Sun. No candidate objects were found in a total of 384 stars.
\end{abstract}

\section{Introduction}

We have shown that Dyson (1960) spheres associated with solar-type stars can be detected as the excess flux at $12 \mu$ (Jugaku \& Nishimura 1991). Even if only $10^{-2}$ of the radiation energy of the central star is converted to thermal emission from a partial (incomplete) Dyson sphere maintained near $300 \mathrm{~K}$, it could be easily detected with the present capability of infrared photometry. In particular, we note that $2.2 \mu$ radiation ( $K$ magnitude) is a good measure of the photospheric radiation of a central star. Then, the index $K-[12]$, where [12] denotes the magnitude at $12 \mu$, should show significant positive values for Dyson spheres.

\section{Observational Material}

The catalog compiled by Woolley et al. (1970) lists 1,744 star systems nearer than $25 \mathrm{pc}$. We find 887 stars of spectral types $\mathrm{F}, \mathrm{G}$, and $\mathrm{K}$ and luminosity classes IV, V, and VI in the catalog, out of which 458 stars are also listed in the IRAS Point Source Catalog (1988) as measured at $12 \mu$. In Papers I - III (Jugaku et al. 1995; Jugaku \& Nishimura 1997; 2000) we examined $K$ - [12] color of 365 solar-type stars from those 458 stars. In this continued study, we examine $K-[12]$ index for 19 stars by utilizing the recently released data of the Two-Micron All-Sky Survey (2MASS). The results are summarized in Table 1.

\section{Conclusion}

Combined with the results of Paper I (180 stars), Paper II (50 stars), and Paper III (135 stars), we have not found any candidate stars which may have Dyson spheres in a sample of 384 solar-type stars within 25 pc of the Sun.

Acknowledgments. Information retrieval for this study was supported by facilities of the Astronomical Data Analyis Center of the National Astronomical Observatory, Japan. 
Table 1. The first column gives the star number in the Woolley et al. (1970) catalog, the second column shows the HD number, the third column is the spectral type from Woolley et al. (1970), the fourth column is $K$ magnitude taken from 2MASS, and the fifth column gives $K$ - [12] color. For all stars the value of $K-[12]$ color is in the range of -0.30 to $+0.30 \mathrm{mag}$. The scatter in this range can be explained by observational errors. If the waste heat of a Dyson sphere is in the order of $10^{-2}$ of the radiation energy of the central star, the color excess should be $>1$ mag.

\begin{tabular}{ccccc}
\hline RGO Star No. & HD & Sp. Type & $K(\mathrm{mag})$ & $K-[12](\mathrm{mag})$ \\
\hline 42 & 5133 & K3 & 4.89 & -0.24 \\
$60 \mathrm{~A}$ & 9770 & K3 & 4.68 & 0.05 \\
103 & 16157 & K7 & 4.89 & 0.22 \\
142 & 21531 & K7 & 5.08 & 0.29 \\
$157 \mathrm{~A}$ & 24916 & K5 & 5.36 & 0.19 \\
$200 \mathrm{~A}$ & 34673 & K3 & 5.01 & 0.11 \\
201 & 35171 & K5 & 5.22 & 0.22 \\
204 & 36003 & K5 & 4.87 & 0.10 \\
259 & 52698 & K0 & 4.62 & 0.02 \\
260 & 53143 & K0 & 4.98 & -0.04 \\
$9409 \mathrm{~A}$ & 108799 & F8 & 4.85 & 0.16 \\
481 & 110315 & K8 & 5.07 & 0.14 \\
529 & 120467 & K6 & 5.16 & 0.06 \\
$593 \mathrm{~A}$ & 139341 & K4 & 4.71 & 0.04 \\
614 & 145675 & K1 & 4.69 & 0.09 \\
9613 & 164922 & K0 & 5.10 & -0.11 \\
$859 B$ & 212697 & G1 & 4.10 & 0.12 \\
868 & 214749 & K5 & 5.04 & 0.21 \\
886 & 217580 & K4 & 5.22 & -0.02 \\
\hline
\end{tabular}

\section{References}

Dyson, F. J. 1960, Science, 131, 1967

Infrared Astronomical Satellite (IRAS) Catalogs and Atlases 1988, Vols. 1-7, NASA RP-1190, (Washington D.C.: NASA)

Jugaku, J., \& Nishimura, S. 1991, in Bioastronomy: The Search for Extraterrestrial Life, ed. J. Heidmann \& M. J. Klein, (Berlin: Springer), 295

Jugaku, J., Noguchi, K. \& Nishimura, S. 1995, in ASP Conf. Ser. 74, Progress in the Search for Extraterrestrial Life, ed., G. S. Shostak, 381 (Paper I)

Jugaku, J. \& Nishimura, S. 1997, in Astronomical and Biochemical Origins and the Search for Life in the Universe, ed. C. B. Cosmovici, S. Bowyer, \& D. Werthimer, (Bologna: Editrice Compositori), 707 (Paper II)

Jugaku, J. \& Nishimura, S. 2000, in ASP Conf. Ser. 213, Bioastronomy '99: A New Era in the Search for Life, ed. G. A. Lemarchand \& K. J. Meech, 213, 581 (Paper III)

Woolley, R., Epps, E. A., Penston, M. J., \& Pocock, S. 1970, Roy. Obs. Ann., 5 